\title{
Flow induced vibration of corrugated rod subjected to axial flow
}

\author{
Y. Perets ${ }^{1}$, B. Rivin ${ }^{2}$, I. Bronstein ${ }^{2}$, R. Harari ${ }^{1}$ \& E. Sher ${ }^{2}$ \\ ${ }^{I}$ Nuclear Research Center - Negev (NRCN), Israel \\ ${ }^{2}$ Ben-Gurion University of the Negev, Israel
}

\begin{abstract}
Preliminary experiments were performed for studying flow induced vibration of a rod wrapped by transverse shapes corrugation (repeated ribs) and subjected to axial flow. Flexible rods with different repeated ribs pitches and widths were tested. The vibration amplitude was measured and the damping ratio of every rod was evaluated, in order to estimate the amplification effect of the repeated ribs on the rod, compared to the results on a smooth rod. The effect of different parameters on the vibration is being discussed. It was found that the repeated ribs have an effect on the pressure drop increases produced by the turbulence augmentations. Empirical correlations form of the vibration amplitude at middle rod span is being proposed.
\end{abstract}

Keywords: flow induced vibration, corrugation, repeated ribs.

\section{Introduction}

Corrugation structures or repeated ribs are well known as a heat transfer enhancement technique in various engineering applications such as: heat exchanger, insert technology, air conditioning and refrigeration systems, chemical reactors, fuel rod and control rod components.

In the literature the repeated ribs are treated as a roughness geometry problem or as a boundary layer separation and reattachment problem. The repeated ribs create local wall turbulence due to the flow separation and reattachment between the ribs which enhance the heat transfer. At the same time, turbulence is accompanied by increases of pressure fluctuations and flow resistance. Various studies have been preformed in order to evaluate the friction coefficient of repeated ribs roughness (Lewis [1]; Wilkie et al. [2]; Webb et al. [3]). The ribs were characterized by geometric parameters: pitch (p), width (b) and height (e). 
Webb et al. [3] summarize the flow patterns downstream from the ribs as a function of characteristic dimensionless parameter of relative rib spacing ( $\mathrm{p} / \mathrm{e})$. The rib causes the separation, forming a shear layer which reattaches the structure surface at 6 to 8 times the rib height downstream, known as the separation length. In less than 6e reattachment does not occur.

The aforementioned phenomenon influences upon the forces surrounding the rod's structures and can increase its dynamic response. This study has been conducted to examine if the transverse repeated ribs has any effect on the rod vibration in annular flow geometry, where the rod surface is the inner annulus surface.

\section{Experimental apparatus}

Perspex rods with different $\mathrm{p} / \mathrm{e}$ and $\mathrm{b}$ ribs parameters were examined, see Figure 1 . The rods were vertically mounted, clamped at the upper end and freed at the other end. The parameters are summarized in Table 1. The rods' vibrations (amplitude and frequencies of rod motion) were visually monitored using a high speed camera.

Table 1: $\quad$ Rods parameters

\begin{tabular}{|l|c|c|c|}
\hline Parameters & Units & Rod No. 1 & Rod No. 2 \\
\hline L - Rod Length & $\mathrm{mm}$ & 1000 & 1000 \\
\hline$\beta$ - Rod mass ratio & $\mathrm{kg} / \mathrm{m}$ & 0.19 & 0.19 \\
\hline EI & $\mathrm{N} \cdot \mathrm{m}^{2}$ & 1.432 & 1.432 \\
\hline $\mathrm{d}_{\text {out }}$ - Rod outside diameter & $\mathrm{mm}$ & 10 & 10 \\
\hline $\mathrm{D}-$ Tube inner diameter & $\mathrm{mm}$ & 16 & 16 \\
\hline $\mathrm{p}$ - Pitch & $\mathrm{mm}$ & 6 & 9 \\
\hline e - Rib height & $\mathrm{mm}$ & 1 & 1 \\
\hline b - Rib width & $\mathrm{mm}$ & 2 & 3 \\
\hline
\end{tabular}

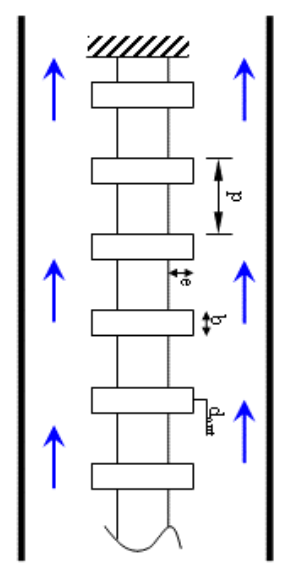

Figure 1: Rod configuration. 


\section{Dimensionless parameters: discussion}

The experiment aim was to examine the influence extent of the transverse repeated ribs on the rod vibration. For this purpose, the pitch and width of the warp ribs were changed, yet no change was performed in the other geometric parameters and the rod properties.

Mass ratio $(\beta)$ : The mass per length ratio has a moderated effect on slender cylinder stability (Païdoussis $[4,5]$ ). In this experiment the mass ratio of the rods was kept constant by increasing the pitch and width of the ribs simultaneously. The mass ratio is:

$$
\beta=\frac{\rho A}{(m+\rho A)}
$$

where $\mathrm{m}$ is the rod mass per unit length; $\rho$ is the fluid density; $\mathrm{A}$ is the crosssectional area of the rod.

Frequency of exciting force: Local pressure fluctuations on the wall due to flow separation and reattachment between the ribs have a characteristic frequency. This frequency can be expressed by ratio of the flow velocity and pitch between the ribs (the same as the pitch between the two local separation and reattachment points). Even at repeated ribs where $\mathrm{p} / \mathrm{e}<6 \mathrm{e}$ and reattachment does not occur, there exist the same characteristic frequency correspondence to the separation point only. The dimensionless frequency is:

$$
\bar{f}=\frac{u}{p \cdot f_{\Omega}}
$$

where $f_{\Omega}$ is the frequency of the rod motion.

Effect of $\varepsilon$ : The effect of $\mathrm{L} / \mathrm{d}_{\text {in }}$ ratio on the stability of the flexible cylinder is obvious the vibration amplitude increases with $\varepsilon$. Nevertheless, increasing of $\varepsilon$ hinders the instability mode. This means that the flow velocity for buckling or fluttering gets larger and at certain values of $\varepsilon$ the first and second mode (buckling and flutter) vanish (Païdoussis [5]).

Reynolds number: The effect of the flow velocity on the rod vibration can be represented by Reynolds number - Re, which expresses the extent of the flow turbulence.

Damping factor: Fluid damping is generated as the rod vibrates normal to the flow. The fluid damping is proportional to rod velocity. Generally, damping increases linearly with the velocity increase (Chen [6]).

In this study the damping factor was follows Fritz [7] model for frictional pressure drop. The friction pressure drop of the annular tube (filled with the transverse corrugation rod) was based on Darcy's friction factor; so the pressure drop was measured as function of the flow velocities. The frictional energy was compared to the energy of fluid viscose damping $(b \cdot u)$ yield:

$$
\zeta=\frac{f_{r} u}{3 \pi^{2} D_{H} f_{1}}
$$


where $\mathrm{u}$ is the mean flow velocity, $\mathrm{D}_{\mathrm{H}}=\mathrm{D}_{\text {out }}-\mathrm{D}_{\mathrm{eq}}$, and $\mathrm{D}_{\mathrm{eq}}$ is the average diameter of the inside and outside diameter of the rod and $f_{l}$ is the rod first mode natural frequency for clamp-free boundary conditions.

The rib characteristic $-\gamma: \gamma=\mathrm{p} / \mathrm{e}$ indicates the flow patterns and the flow separation length. In a case of a smooth rod surface (non repeated ribs), the "pitch" was considered as the rod length (L).

\section{Preliminary results}

The rod vibration amplitude was measured in the middle of the rod length for a number of flow velocities. The dimensionless amplitude of rods vibration contains different transverse repeated ribs versus Reynolds number are plotted in Figure 2. The Reynolds number range, based on test-section hydraulic diameter was $1.1 \times 10^{4}-6.2 \times 10^{4}$. The amplitude of a $10 \mathrm{~mm}$ diameter smooth surface rod is also shown for comparison.

Generally, the amplitude increases with flow velocity increase. For the smooth rod and ribs rod with $\mathrm{p} / \mathrm{e}=6$ the increase is moderated and for the rod with $\mathrm{p} / \mathrm{e}=9$ ribs the increase with flow is at a much higher rate. At low Reynolds number the increase is moderate, but the amplitude increase became steeper at $\operatorname{Re} \approx 22,000$.

From these results we can deduce that the repeated ribs have an amplification effect on the rods motion. As p/e increased the amplitude was increasing too. It is suggested that vibration amplification is induced by pressure fluctuation augmentation due to separation and reattachment between those ribs. For the p/e $=6$ rod no separation occurred and the repeated ribs had a small effect on the rod motion.

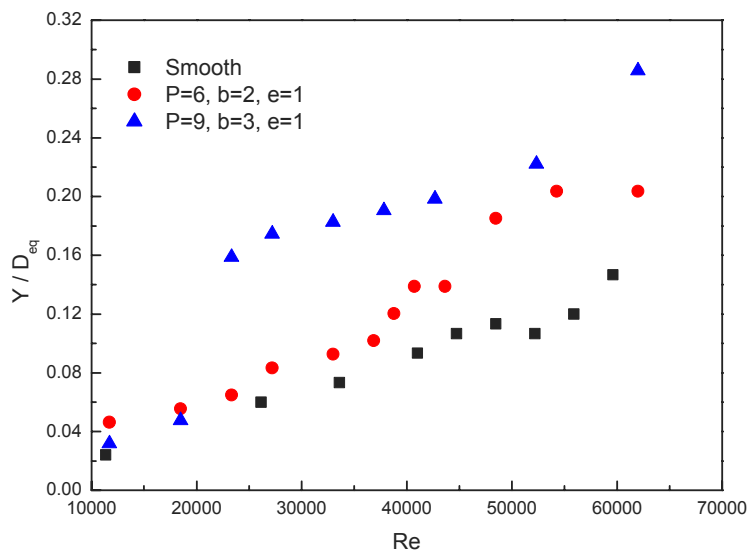

Figure 2: $\quad$ Dimensionless vibration amplitude vs. Re. 


\section{Empirical expression}

The selected dimensionless parameters used for the empirical expression were given in previous section on the dimensionless parameters discussion. A multiple form a multiple form to describe the empirical expression of the amplitude vibration is being suggested.

$$
\frac{Y}{D_{e q}}=a 1 \cdot \varepsilon^{a 2} \beta^{a 3} \operatorname{Re}^{a 4} \gamma^{a 5} \bar{f}^{a 6} \zeta^{a 7}
$$

where $a_{1}$ through $a_{7}$ are dimensionless constants to be found by a nonlinear regression analysis of the experimental data.

Since we preformed only a few numbers of experiments and only one parameter was varied $(\mathrm{p} / \mathrm{e})$ while all other parameters were kept constant, we did not obtain the constant values for eqn (4).

\section{Conclusions}

The presented results support the assumption that repeated ribs have an amplification effect on the dynamic response of the rod. The vibration amplification is stimulated by the pressure fluctuation augmentation due to separation and reattachment between those ribs. A great number of experiments should be conducted with a large range of parameters in order to obtain correlation fit with a good overall agreement.

\section{References}

[1] Lewis, M. J., Optimizing the Thermohydraulic Performance of Rough Surfaces. Int. J. Heat Mass Transfer, 18, pp. 1243 - 1248, 1975.

[2] Wilkie, D., Cowin, M., Burnett, P. \& Burgoyne, T., Friction Factor Measurements in a Rectangular Channel with Walls of Identical and NonIdentical Roughness. Int. J. Heat Mass Transfer, 10, pp. 611 - 621, 1967.

[3] Webb, R. L., Eckert, E. R. G. \& Goldstein, R. J., Heat Transfer and Friction in Tubes with Repeated-Rib Roughness. Int. J. Heat Mass Transfer, 14, pp. $601-617,1971$.

[4] Païdoussis, M.P., Dynamics of Flexible Slender Cylinders in Axial Flow, Part 1.Theory. J. Fluid Mech., 26(4), pp. 717 - 736, 1966.

[5] Païdoussis, M.P., Dynamics of Flexible Slender Cylinders in Axial Flow, Part 2. Experiments. J. Fluid Mech., 26(4), pp. 737 - 751, 1966.

[6] Chen, S. S., Fluid Damping for Circular Cylinder Structure. Nuclear Engineering and Design, 63, pp. 81 - 100, 1981.

[7] Fritz, R. J., The Effect of Liquids Dynamic Motions of Immersed Solids. Journal of Engineering for Industry, 94, pp. 167 - 173, 1972. 